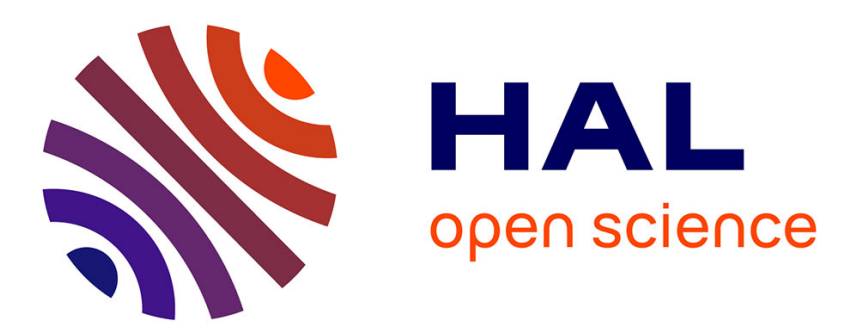

\title{
Transient dynamics and stability of keyhole at threshold in laser powder bed fusion regime investigated by finite element modeling
}

Yaasin A. Mayi, Morgan Dal, Patrice Peyre, Michel Bellet, Charlotte Metton, Clara Moriconi, Remy Fabbro

\section{To cite this version:}

Yaasin A. Mayi, Morgan Dal, Patrice Peyre, Michel Bellet, Charlotte Metton, et al.. Transient dynamics and stability of keyhole at threshold in laser powder bed fusion regime investigated by finite element modeling. Journal of Laser Applications, 2021, 33 (1), pp.1-10. 10.2351/7.0000330 . hal-03160606v2

\section{HAL Id: hal-03160606 https://hal.science/hal-03160606v2}

Submitted on 5 Mar 2021

HAL is a multi-disciplinary open access archive for the deposit and dissemination of scientific research documents, whether they are published or not. The documents may come from teaching and research institutions in France or abroad, or from public or private research centers.
L'archive ouverte pluridisciplinaire HAL, est destinée au dépôt et à la diffusion de documents scientifiques de niveau recherche, publiés ou non, émanant des établissements d'enseignement et de recherche français ou étrangers, des laboratoires publics ou privés. 


\title{
Transient dynamics and stability of keyhole at threshold in laser powder bed fusion regime investigated by finite element modeling
}

\author{
Yaasin A. Mayi, ${ }^{1,2}$ Morgan Dal, ${ }^{2}$ Patrice Peyre, ${ }^{2}$ Michel Bellet, ${ }^{3}$ Charlotte Metton, ${ }^{1}$ Clara Moriconi, \\ and Remy Fabbro ${ }^{2}$ \\ AFFILIATIONS \\ ${ }^{1}$ Safran Additive Manufacturing, A Technology Platform of Safran Tech, Rue des Jeunes Bois, Chateaufort, \\ 78114 Magny-Les-Hameaux, France \\ ${ }^{2}$ Laboratoire PIMM, Arts et Metiers Institute of Technology, CNRS, Cnam, HESAM University, 151 boulevard de l'Hopital, 75013 Paris, \\ France \\ ${ }^{3}$ MINES ParisTech, PSL Research University, CEMEF - Centre de Mise en Forme des Matériaux, CNRS UMR 7635, CS10207 rue \\ Claude Daunesse, 06904 Sophia Antipolis Cedex, France
}

\begin{abstract}
A Finite element model is developed with a commercial code to investigate the keyhole dynamics and stability at keyhole threshold, a fusion regime characteristic to laser microwelding or to Laser Powder Bed Fusion. The model includes relevant physics to treat the hydrodynamic problems-surface tension, Marangoni stress, and recoil pressure-as well as a self-consistent ray-tracing algorithm to account for the "beam-trapping" effect. Implemented in both static and scanning laser configurations, the model successfully reproduces some key features that most recent $\mathrm{x}$-ray images have exhibited. The dynamics of the liquid/gas interface is analyzed, in line with the distribution of the absorbed intensity as well as with the increase of the keyhole energy coupling. Based on these results, new elements are provided to discuss our current understanding of the keyhole formation and stability at threshold.
\end{abstract}

Key words: laser-matter interaction, keyhole, beam trapping, multiple reflections, keyhole instabilities, laser welding

\section{INTRODUCTION}

Laser Powder Bed Fusion (LPBF) has become an extremely attractive additive manufacturing process for industrial applications because it is suited to produce small to medium components $(\sim 1-50 \mathrm{~cm})$ of high complexity and high added value. Basically, a laser source of a few hundred watts and a small focal spot $(\sim 100 \mu \mathrm{m})$ is used to melt at high velocity $(\sim 1 \mathrm{~m} / \mathrm{s})$ a powder bed together with its building plate or some previously solidified layers (Fig. 1). Although manufacturing suitable LPBF parts is determined by more than 130 processing parameters, ${ }^{1}$ at the local scale and at a given working environment, melt pool hydrodynamics is primarily affected by the combination of the material properties, laser power, laser velocity, and spot diameter. ${ }^{2}$ Recent $\mathrm{x}$-ray investigations have shown that the presence of powder bed has only a secondary-order effect on keyhole formation mechanisms, ${ }^{3}$ resulting, however, in more process variability than in the bare plate configuration. Hence, to some extent, LPBF may be analyzed as a laser microwelding process with powder feedstock. From this point of view then, many of the issues encountered in laser welding have been brought up to date with the LPBF process.

One of the critical issues to implement laser welding processes or LPBF is to understand the physical bases of keyhole formation and stability. This thematic has been extensively investigated, primarily by high speed imaging, ${ }^{4,5}$ sometimes combined with in situ observation setups based on transparent model materials such as water, ice, or glycerin, ${ }^{6,7}$ and is increasingly relying on dynamic 


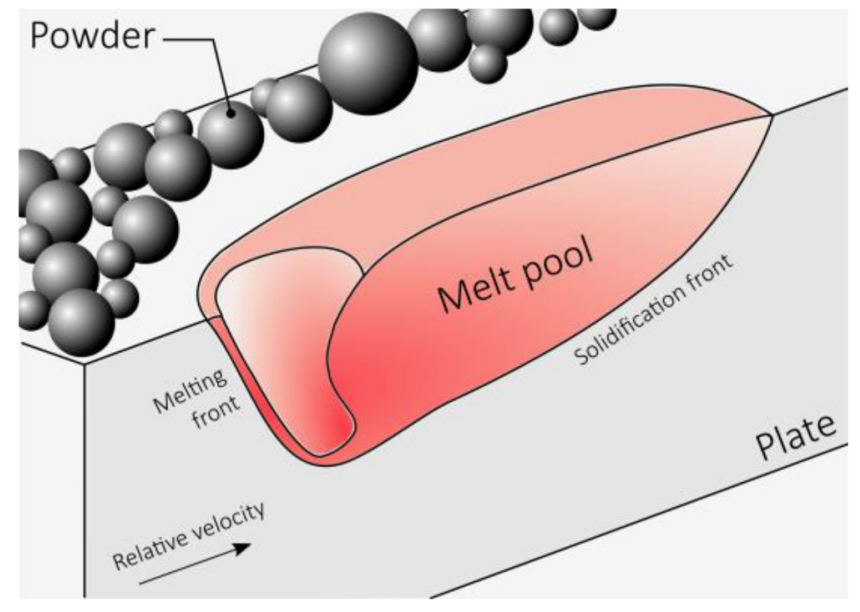

FIG. 1. Schematics of the melt pool in LPBF.

x-ray imaging. ${ }^{3,8-12}$ Such experimental hindsight shows that keyhole formation is deeply related to the multiple reflections of the incident irradiation in the vapor depression. When the vapor depression reaches a critical aspect ratio, the incident radiation is reflected toward the melt pool instead of being deflected outward and get somewhat "trapped." Recent microcalorimetric measurements of melt pool absorptance ${ }^{13}$ confirm that the melt pool energy coupling sharply increases at the conduction-to-keyhole transition. Additionally, integrating sphere measurements ${ }^{14}$ have revealed that the keyhole absorptance fluctuates over time, particularly at the onset of keyhole formation. Such fluctuations are believed to reflect the keyhole geometry oscillations, which have been pointed out for a long time either by direct melt pool observation or indirectly by capturing the oscillating signals emitted by the vapor plumes. ${ }^{8,15}$

To better understand such mechanisms and to access quantitative information that are difficult to acquire experimentally, the previous issues are for the past 20 years, increasingly investigated by numerical modeling. Some multiphysical models account for the "beam trapping" effect indirectly by increasing artificially the material absorptance. ${ }^{16,17}$ Others account for this phenomenon self-consistently often by integrating a ray-tracing (RT) algorithm in their model ${ }^{18-25}$ or less frequently by solving Maxwell's equations. ${ }^{26}$ Early works such as those of Ki et al. ${ }^{18}$ and of Lee et al. ${ }^{19}$ have investigated the link between the "beam trapping" effect, the keyhole energy coupling, and the melt pool stability in the spot welding configuration. They have looked at the intensity redistribution on the keyhole walls, due to the beam multireflections and to the melt pool corrugations. More recently, Kouraytem et al..$^{25}$ gave a quite complete picture of the laser welding keyhole dynamics around the steady state, as well as an analysis of the protrusions dynamics which form at the keyhole front wall. Moreover, contrary to most previous studies on the same subject, their model has been duly validated, thanks to dynamic $\mathrm{x}$-ray images.

The objective of the present work is to give a complete analysis of the transient keyhole formation process, with a focus on the mechanisms that lead to its fluctuations at threshold. To do so, a
Finite Element (FE) model is developed with COMSOL MULTIPHYSICS ${ }^{\oplus}$ The code includes the melt pool hydrodynamic (surface tension, Marangoni stress, and recoil pressure) as well as a self-consistent RT algorithm to account for the "beam trapping" effect. The model is then used to reproduce published experiments, to validate the modeling approach, and to investigate the complex coupling between the optical and hydrodynamic phenomena that drive keyhole formation.

\section{COMPUTATIONAL MODEL}

\section{A. Geometrical configuration}

As explained in Sec. I, the powder bed has only a secondaryorder effect on keyhole formation mechanisms. ${ }^{3}$ For this reason, the following simulations are performed without including the powder bed, for simplification purpose.

\section{B. Laser beam energy deposition}

The laser heat source is computed using the RT approach. The incident laser beam is discretized into $N_{\text {ray }}$ individual rays that carry a fraction of the incident power and interact with the material in accordance with the law of geometrical optics. The incident laser flux is modeled by a Gaussian law, typical to the fundamental $\mathrm{TEM}_{00}$ mode,

$$
\vec{\varphi} \text { laser }=\frac{2 P}{\pi R_{0}^{2}} \exp \left(-2 \frac{r^{2}}{R_{0}^{2}}\right) \vec{k},
$$

where $P$ and $R_{0}$ are, respectively, the laser power and the $1 / e^{2}$ spot radius, $r$ is the radial distance to the laser axis, and $\vec{k}$ is the direction of ray propagation.

Then, the RT algorithm is designed to calculate the absorbed laser intensity in accordance with the keyhole geometry (Fig. 2). At the first laser-material interaction, prior to any multiple reflections, the absorbed intensity $I_{\mathrm{abs}}^{\mathrm{i}}$ is initialized analytically as

$$
I_{\mathrm{abs}}^{\mathrm{i}}=\left[1-R_{F}\right] \vec{\varphi} \text { laser } \cdot \vec{n},
$$

where $R_{F}$ is the reflectivity and $\vec{n}$ is the outer normal to the metal surface.

Thereafter, as the melt pool depression forms, there is a point from which it is necessary to account for the multireflected rays in the local absorbed intensity,

$$
I_{\mathrm{abs}}=\sum_{\text {ray }=1}^{N_{\text {ray }}} \sum_{\text {refl }=1}^{N_{\text {refl }}}\left[1-R_{F}\right] \vec{\varphi} \text { laser } \cdot \vec{n},
$$

where $N_{\text {refl }}$ is the number of reflections per ray.

More details about the algorithm are given in Ref. 27.

\section{Heat transfer}

Temperature field is computed by solving the transient heat conservation equation

$$
\rho c_{p}^{e q} \frac{\partial T}{\partial t}+\rho c_{p}(\vec{u} \cdot \vec{\nabla} T)=\vec{\nabla} \cdot(k \vec{\nabla} T)
$$




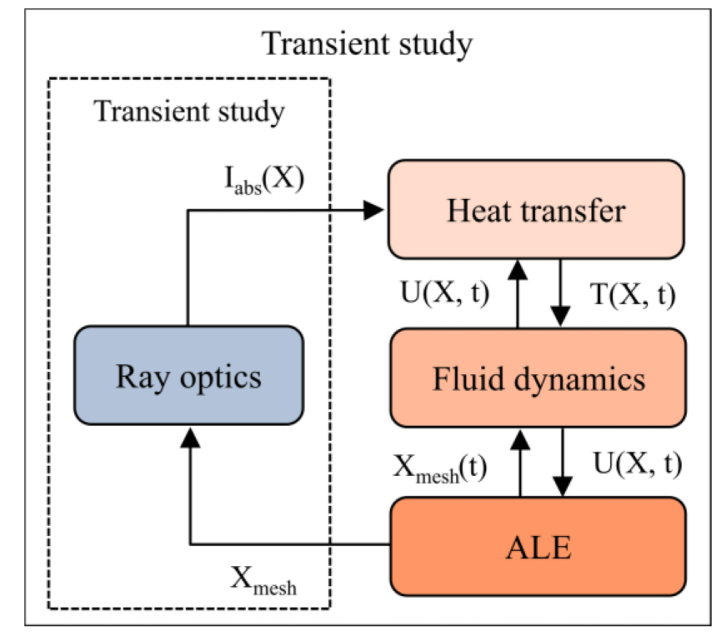

FIG. 2. Schematics of laser heat source update strategy. Adapted from M. Courtois, M. Carin, P. Le Masson, S. Gaied, and M. Balabane, "Complete heat and fluid flow modeling of keyhole formation and collapse during spot laser welding," in International Congress on Applications of Lasers \& Electro-Optics, Miami, FL, USA (Laser Institute of America, 2013), pp. 77-84.

where $c_{p}$, $k$, and $\rho$ stand for the specific heat, the thermal conductivity, and the density, respectively.

The enthalpy of fusion $L_{m}$ is accounted for through an equivalent specific heat,

$$
c_{p}^{\mathrm{eq}}=c_{p}+\frac{L_{m}}{\sqrt{\pi \Delta T^{2}}} \exp \left[-\frac{\left(T-T_{\mathrm{m}}\right)^{2}}{\Delta T^{2}}\right]
$$

where $\Delta T=\left(T_{\text {liq }}-T_{\text {sol }}\right) / 2$ is the fusion interval and $T_{\mathrm{m}}=\left(T_{\mathrm{liq}}+T_{\mathrm{sol}}\right) / 2$ is the melting temperature.

The laser heat flux is counterbalanced by vaporization losses,

$$
k \vec{\nabla} T \cdot(-\vec{n})=I_{\mathrm{abs}}-\dot{m} L_{\mathrm{v}},
$$

where $L_{V}$ is the latent heat of vaporization.

Heat lost by radiation and convection is neglected compared to vaporization losses. The ablation flux $\dot{m}$ is computed $\mathrm{as}^{29}$

$$
\begin{gathered}
\dot{m}=\sqrt{\frac{M}{2 \pi \mathrm{RT}_{\mathrm{s}}}} P_{\mathrm{sat}}(T)+\beta_{R} \sqrt{\frac{M}{2 \pi R T_{\mathrm{KN}}}} P_{\mathrm{sat}}\left(T_{\mathrm{KN}}\right) f\left(\emptyset_{\mathrm{KN}}\right), \\
f\left(\emptyset_{\mathrm{KN}}\right)=\sqrt{\pi} \emptyset_{\mathrm{KN}} \operatorname{erfc}\left(\emptyset_{\mathrm{KN}}\right)-\exp \left(-\emptyset_{\mathrm{KN}}^{2}\right), \\
\emptyset_{\mathrm{KN}}=\mathrm{Ma}_{\mathrm{Kn}}^{2} \gamma / 2,
\end{gathered}
$$

where $M$ is the molar mass, $\mathrm{Ma}_{\mathrm{KN}}$ and $T_{\mathrm{KN}}$ are, respectively, the Mach number and the temperature outside of the Knudsen layer, $\beta_{R}$ is the retro-diffusion coefficient, and $\gamma$ is the heat capacity ratio.

$\mathrm{Ma}_{\mathrm{KN}}$ and $T_{\mathrm{KN}}$ are determined according to Knight's method $^{30}$ and the saturated vapor pressure $P_{\text {sat }}$ is calculated, thanks to the Clausius-Clapeyron law,

$$
P_{\text {sat }}(T)=P_{\text {atm }} \exp \left[\frac{M L_{v}}{R T_{v}}\left(1-\frac{T_{v}}{T}\right)\right],
$$

where $P_{\text {atm }}$ is the atmospheric pressure and $T_{V}$ is the boiling temperature at atmospheric pressure.

\section{Fluid flow}

Transient mass [Eq. (10)] and momentum [Eq. (11)] balance are computed in their incompressible form,

$$
\vec{\nabla} \cdot \vec{u}=0
$$

$$
\rho \frac{\partial \vec{u}}{\partial t}+\rho(\vec{u} \cdot \vec{\nabla}) \vec{u}=\vec{\nabla} \cdot\left\{-p I+\mu\left[\vec{\nabla} \vec{u}+(\vec{\nabla} \vec{u})^{\mathrm{T}}\right]\right\}+\vec{f}_{v}
$$

where $\mu$ is the viscosity of the liquid phase.

The bulk force $\vec{f}_{v}$ is a Darcy's penalization term used to model the solid/liquid transition, ${ }^{31}$

$$
\vec{f}_{v}=-C_{1} \frac{\left(1-f_{\text {liq }}\right)^{2}}{f_{\text {liq }}^{3}+C_{2}} \vec{u},
$$

where $f_{\text {liq }}$ is the liquid fraction and $C_{1}$ and $C_{2}$ are two numerical constants tailored to penalize velocity in the solid.

Stress balance at the liquid/gas interface gives

$$
\begin{aligned}
\left(-p I+\mu\left[\vec{\nabla} \vec{u}+(\vec{\nabla} \vec{u})^{\mathrm{T}}\right]\right) \cdot \vec{n}= & -\left(P_{s}-P_{\mathrm{atm}}\right) \cdot \vec{n}+\sigma \kappa \vec{n} \\
& +\frac{\partial \sigma}{\partial T} \vec{\nabla}_{S} T,
\end{aligned}
$$

where $\sigma$ is the surface tension coefficient and $\kappa$ is the liquid/gas interface curvature.

As for the ablation flux, a complete expression of the recoil pressure is derived as ${ }^{29}$

$$
\begin{gathered}
P_{s}=\frac{1}{2} P_{\mathrm{sat}}\left(T_{s}\right)+\beta_{R} P_{\mathrm{sat}}\left(T_{\mathrm{KN}}\right) g\left(\emptyset_{\mathrm{KN}}\right), \\
g\left(\emptyset_{\mathrm{KN}}\right)=\left[\left(\emptyset_{\mathrm{KN}}^{2}+\frac{1}{2}\right) \operatorname{erfc}\left(\emptyset_{\mathrm{KN}}\right)-\frac{\emptyset_{\mathrm{KN}}}{\sqrt{\pi}} \exp \left(-\emptyset_{\mathrm{KN}}^{2}\right)\right] .
\end{gathered}
$$

\section{E. Interface tracking}

The liquid/gas interface is tracked using the Arbitrary Lagrangian Eulerian method. The interface is discretized with a conformed mesh, and its velocity $V_{I}$ follows the fluid movement according to

$$
V_{\mathrm{I}}=\vec{u} \cdot \vec{n} .
$$

Interface displacement is then propagated through the whole domain, following the Yeoh smoothing $\operatorname{method}^{32}$ to ensure a smooth mesh deformation. 
TABLE I. Material properties and numerical parameters.

\begin{tabular}{|c|c|c|c|}
\hline \multicolumn{2}{|c|}{ Thermophysical properties (units) } & \multirow{2}{*}{$\begin{array}{c}\text { Values } \\
740\end{array}$} & \multirow{2}{*}{$\frac{\text { Referenc }}{33}$} \\
\hline$c_{p}$ & Specific heat $(\mathrm{J} / \mathrm{kg} \mathrm{K})$ & & \\
\hline$k^{P}$ & Thermal conductivity $(\mathrm{W} / \mathrm{m} \mathrm{K})$ & 26 & 33 \\
\hline$k_{F} / n_{F}$ & Refractive indexes of $\mathrm{Ti}$ & $4.0 / 3.5$ & 34 \\
\hline$L_{m}$ & Enthalpy of melting $(\mathrm{J} / \mathrm{kg})$ & $2.86 \times 10^{5}$ & 33 \\
\hline$L_{v}$ & Enthalpy of vaporization $(\mathrm{J} / \mathrm{kg})$ & $8.90 \times 10^{6}$ & 35 \\
\hline$M$ & Molar mass $(\mathrm{g} / \mathrm{mol})$ & 0.0479 & 33 \\
\hline$T_{\text {sol }}$ & Solidus temperature $(\mathrm{K})$ & 1878 & 33 \\
\hline$T_{\text {liq }}$ & Liquidus temperature $(\mathrm{K})$ & 1923 & 33 \\
\hline$T_{v}$ & Boiling temperature $(\mathrm{K})$ & 3558 & 35 \\
\hline$\gamma$ & Heat capacity ratio & 1.67 & - \\
\hline$\mu$ & Dynamic viscosity (Pa s) & 2.0 & 33 \\
\hline$\rho$ & Density $\left(\mathrm{kg} / \mathrm{m}^{3}\right)$ & 4200 & 33 \\
\hline$\Sigma$ & Surface tension $(\mathrm{N} / \mathrm{m})$ & 1.38 & 36 \\
\hline$\partial \sigma / \partial T$ & Thermocapillary coeff. (N/m K) & $-0.31 \times 10^{-3}$ & 36 \\
\hline \multicolumn{4}{|c|}{ Numerical constants } \\
\hline$C_{1} / C_{2}$ & Penalization constants & $10^{6} / 10^{-5}$ & - \\
\hline$N_{\text {ray }}$ & Number of rays & 50000 & - \\
\hline
\end{tabular}

\section{F. Properties and numerical constants}

The material under investigation is the titanium alloy Ti-6-Al-4V. Constant indicative property values are summarized in
Table I. In the model, notice that full temperature-dependent properties are implemented, using the data compiled in Refs. 33-36.

\section{G. Numerical setup}

This model is implemented in the commercial FE software COMSOL MULTIPHYSics ${ }^{\oplus} 5.5,{ }^{32}$ using the "developer" mode. All details of the numerical setup are provided in Ref. 27.

\section{EXPERIMENTAL DATA}

The authors use Cunningham's experimental results ${ }^{3}$ as reference data to validate the present FE model. The data are extracted with the software IMAGEJ, and some $\mathrm{x}$-ray images are embedded in the figures.

\section{RESULTS AND DISCUSSION}

\section{A. Keyhole dynamics during stationary illumination}

Stationary laser illumination is simulated, with a laser power of $156 \mathrm{~W}$ and a $1 / e^{2}$ spot size of $140 \mu \mathrm{m}\left(I=1 \mathrm{MW} / \mathrm{cm}^{2}\right)$. This simulation aims to validate the model method with published experimental results and to provide new material that describes keyhole formation mechanisms.

Figures 3(a)-3(e) compare the simulated keyhole formation steps to their experimental counterparts. ${ }^{3}$ For each step, the optical path and the normalized power of the rays are represented to show how optics and hydrodynamics are involved in this process. First,

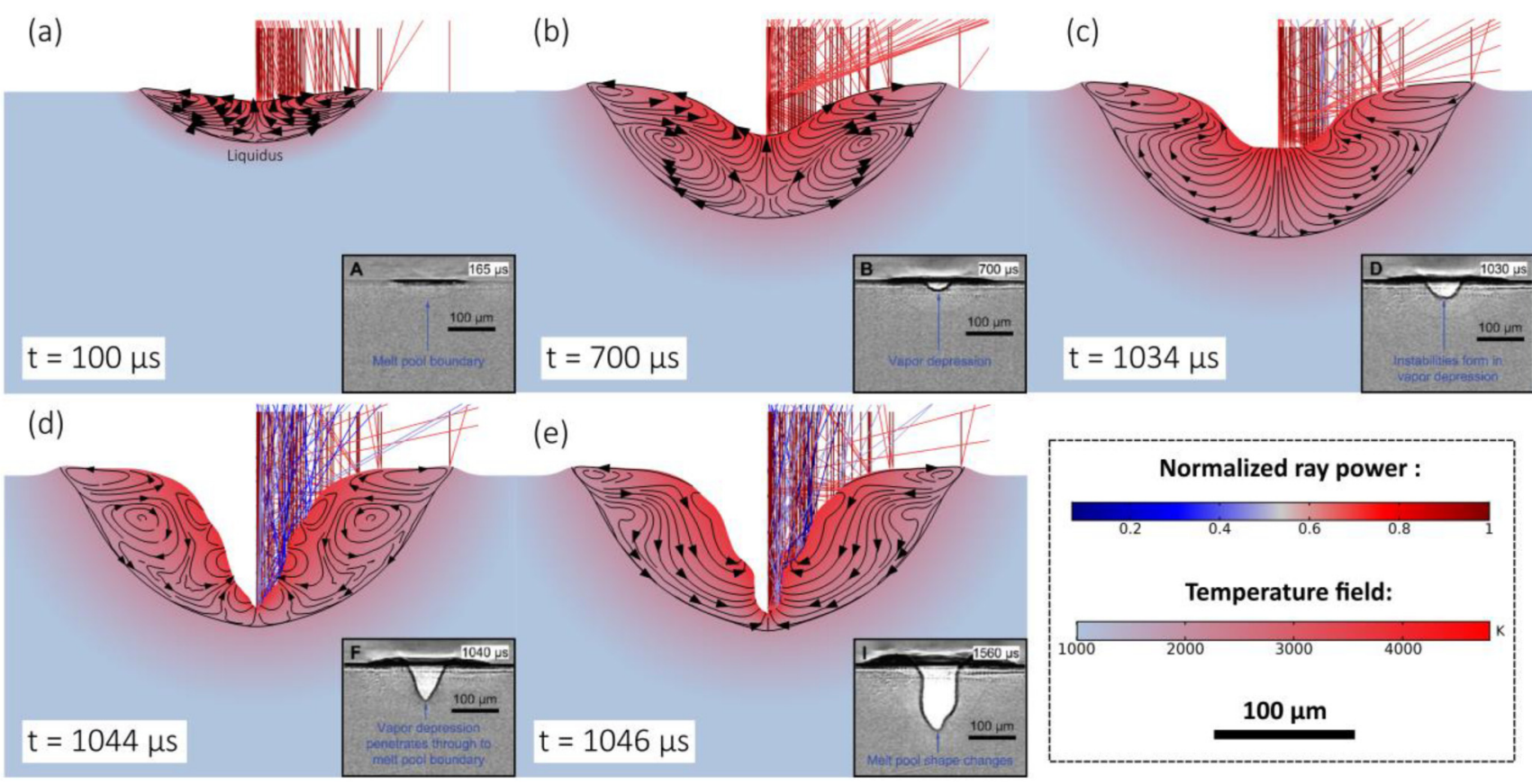

FIG. 3. Keyhole formation steps during stationary irradiation. (a) Conduction mode. (b) Forced conduction mode. (c) Vapor depression instabilities. (d) Keyhole mode. (e) Keyhole fluctuations. X-ray images were reprinted with permission from R. Cunningham, C. Zhao, N. Parab, C. Kantzos, J. Pauza, K. Fezzaa, T. Sun, and A. D. Rollett, Science 363, 849-852 (2019). Copyright 2019, AAAS. 
an evident phenomenological agreement is depicted between the FE and the experimental results. Five steps are identified: (1) conduction melting [Fig. 3(a)], (2) vapor depression formation and growth [Fig. 3(b)], (3) vapor depression instability [Fig. 3(c)], (4) keyhole formation [Fig. 3(d)], and (5) keyhole fluctuations [Fig. 3(e)].

During the conduction step, the melt pool is flat [Fig. 3(a)]. The absorbed intensity is distributed according to the initial Gaussian law (Fig. 4, $t=100 \mu \mathrm{s}$ ), and there is no particular coupling between the optics and the melt pool hydrodynamics. In our simulation, the fraction of incident power absorbed by the melt pool (i.e., the global absorptance) is constant and equal to the material's absorptance at normal incidence-here 0.33 . Physically speaking, the absorptance is in fact likely to increase with temperature and with the eventual formation of oxide layers. ${ }^{37}$ This phenomenon was recently shown by Allen et al. ${ }^{38}$ during stationary illumination experiments but on 316L stainless steel. However, we did not consider these effects here, first because it is difficult to find reliable experimental data on temperature-dependent absorptance, and also for simplification purpose, as we are mainly interested in the next steps where absorption is dominated by laser beam trapping. Anyway, during this stage, the maximum temperature at the liquid interface lies between the liquidus and the boiling temperatures. Hence, Marangoni shear stress-induced by a high temperature gradient of $\sim 5 \times 10^{7} \mathrm{~K} / \mathrm{m}$-is the only driving force, which shears the liquid metal from the center of the interaction zone to the sides of the melt pool (since $\partial \sigma / \partial T<0)$.

Then, when the melt surface temperature reaches and exceeds the boiling point at the center of the interaction zone, vaporization starts. Previous studies have estimated that at similar incident intensities, metal vapor is ejected at several hundred meters per second, ${ }^{39}$ or even at a few thousand meters per second, ${ }^{40}$ depending on the material tested. However, due to the piston effect (i.e., equilibrium between rising plume and surrounding quiescent atmosphere), the plume ascent velocity is 1 order of magnitude lower. ${ }^{39}$ Due to the action-reaction principle, the recoil pressure creates a shallow vapor depression onto the melt pool [Fig. 3(b)], which deepens roughly linearly with time (Fig. 5). Here, the melt flow induced by the recoil pressure is slower than that generated by the Marangoni stress $(\sim 1 \mathrm{~m} / \mathrm{s}$, against $\sim 8 \mathrm{~m} / \mathrm{s}$, respectively). But if the fluid layer sheared by the Marangoni stress is relatively superficial, the recoil pressure literally "drills" the material and ejects the liquid metal from the center to the rim of the melt pool. Consequently, as shown by the well-known "piston" model derived by Semak and Matsunawa, ${ }^{41}$ the recoil pressure becomes the main driving force and $70 \%-90 \%$ of the absorbed power is carried away from the interaction zone by this mechanism. Also, the melt pool keeps its semicircular shape as in the conduction mode, but power lost by conduction represents only about $10 \%$ of the balance. ${ }^{41}$ Furthermore, the vapor depression is not deep enough to trap the incident laser irradiation and the absorbed intensity conserves a Gaussian distribution-weighted by the cosine of the laser incident angle (Fig. $4, t=500 \mu \mathrm{s}$ ). Consequently, as already suggested by Lee et al., ${ }^{19}$ this stage corresponds to an intermediate third melting mode in welding, recently qualified by Fabbro ${ }^{42}$ as a "forced conduction" mode.

Thereafter, the bottom of the vapor cavity starts to oscillate, around a mean value estimated by the dotted red line in Fig. 5, which represents the depression depth calculated without accounting for RT. When the vapor depression gets an aspect ratio of $\sim 0.3-0.4$, a portion of the vaporization front reaches an inclination angle of $\sim 45^{\circ}$, at which the rays are reflected horizontally [Fig. 3(c)]. The absorbed intensity thus locally increases, and its distribution deviates from the initial Gaussian shape. For instance, at $t=1034 \mu \mathrm{s}$, a local intensity maximum (two times higher than that absorbed at the center of the Gaussian) is reached at $r=20 \mu \mathrm{m}$ (Fig. 4). At that location, the temperature exceeds the boiling point and the recoil pressure pushes the vapor front laterally. Consequently, the depression wall is pushed back again above $45^{\circ}$ (relative to the vertical axis) and its depth decreases below the

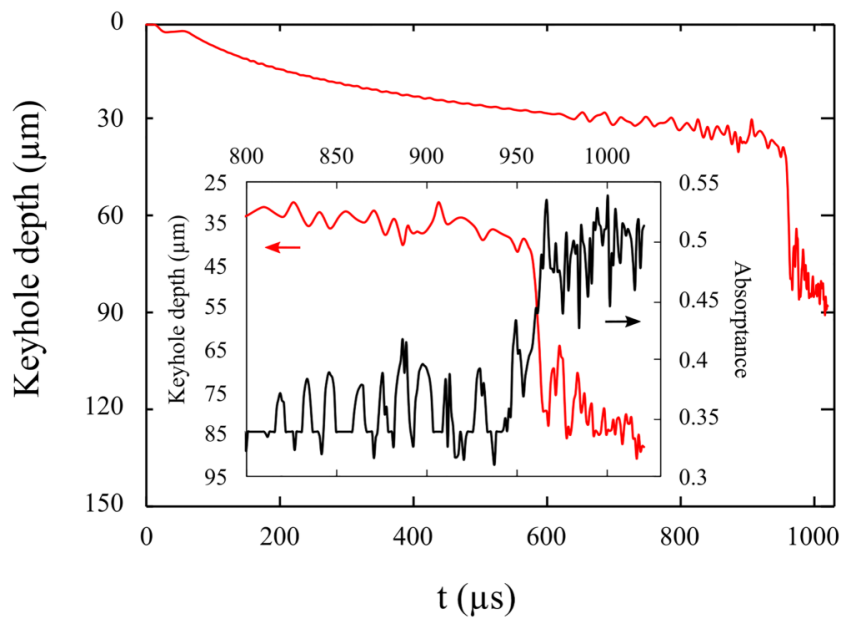

FIG. 5. Keyhole depth (with and without RT) and absorptance over time in the static configuration.
FIG. 4. Distribution of normalized absorbed laser intensity (log scale) at differ-

ent instants.

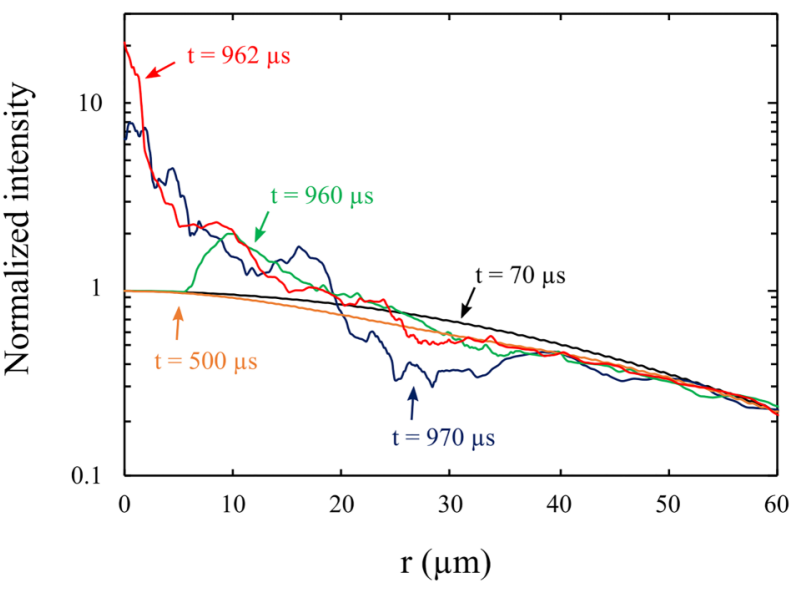


mean value. The absorbed intensity profile then becomes Gaussian again, and the above process repeats periodically, while the vapor depression continues to deepen on average. As the vapor depression oscillates, the global absorptance fluctuates accordingly, from one value theoretically calculated with Fresnel's laws, to another, estimated by Gouffé's law. ${ }^{43}$

However, as the cavity deepens, a critical inclination is reached, at which the incident radiation is reflected downward. Here, this event occurs at $t=1036 \mu$ s. Figure 6 shows how the incident rays are reflected at that time. A fraction of the incident rays (the green ones) are reflected almost horizontally, as they interact with a portion of the vaporization front inclined at $\sim 47^{\circ}$ relative to the vertical axis. These rays leave the cavity after two reflections. However, another fraction (the purple rays) is reflected downward by a portion of the vaporization front inclined at $33^{\circ}$ relative to the vertical axis. These rays then leave the cavity after three reflections. This portion of the melt then acts like a concave mirror, which focuses the laser beam on its optical axis. Consequently, the absorbed intensity sharply increases near the symmetry axis and is multiplied by 20 (Fig. 4, $t=1036 \mu \mathrm{s}$ ). Such a dramatic increase of the absorbed intensity has been also shown by Ki et al. ${ }^{18}$ and is the cause of keyhole formation.

In less than $10 \mu \mathrm{s}$, the semicircular vapor cavity switches into a V-shape keyhole [Fig. 3(d)], $50 \mu \mathrm{m}$ deeper (Fig. 5). In this $\mathrm{V}$-shape keyhole, the incident rays at the center of the beam are reflected three times or more and leave the cavity with a residual power of $\sim 0.1-0.2$ times the incident power [Fig. 3(d)]. The absorbed intensity is completely redistributed on the keyhole wall (Fig. $5, t=1044 \mu \mathrm{s}$ ), and the system is unstable as the incident laser powder is not distributed homogeneously. Consequently, the keyhole loses its initial V-shape, in favor of a chaotic bimodal shape [Fig. 3(e)] as noted by Cunningham et al. ${ }^{3}$ This unstable behavior is apparent not only through the shape of the keyhole but also on both its depth and absorptance that oscillate accordingly. This correlation has been recently experimented by Allen et al., using integrating sphere radiometry and inline coherent imaging.

There are nevertheless some discrepancies between the experiments and the FE results. For instance, the conduction mode is shorter in the model ( $\sim 100$ against $250 \mu \mathrm{s})$ and the predicted melt pool depth over time is overestimated by about $16 \%$. For example, at $t=700 \mu \mathrm{s}$, the predicted melt pool width and depth are,

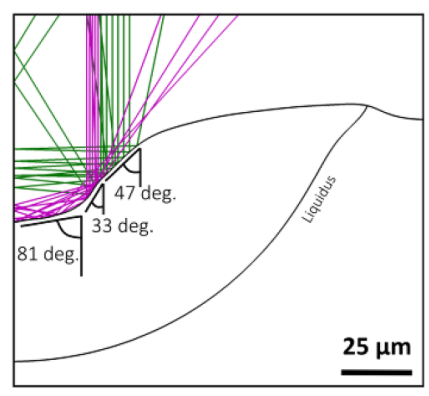

FIG. 6. Keyhole average inclination angles at threshold $(t=1036 \mu \mathrm{s})$. respectively, 226 and $80 \mu \mathrm{m}$, against $\sim 220$ and $\sim 69 \mu \mathrm{m}$ in the experiments. Any model, by definition, results in differences with experiments, often the results of simplifying assumptions. Here particularly, we have used a constant material absorptance. Therefore, the difficulty was to estimate an adequate "average" value, which is supposedly valid on solid and liquid metal, at any temperature. By using Fresnel's law with the refractive indexes of pure $\mathrm{Ti}$, we found a much faster keyhole process than in the experiments. We have therefore lowered the absorptance value from 0.39 to 0.33 to fit the experimental results. Note that recently, Ye et al. ${ }^{24}$ faced the same issue and lowered the absorptance to 0.26 . This challenge illustrates how much works on measurements of material properties are important so that modeling efforts can benefit from reliable material data. Note finally that neglecting absorptance variations with incident angle is not an excessive simplification. As shown in Ref. 42, the induced absorptance variation (before the beam trapping event) is negligible compared to the effect of multireflections. Typically, using the refractive indexes of $\mathrm{Ti}$, it is estimated (assuming the beam is not polarized) that between $90^{\circ}$ and $45^{\circ}$ relative to the vertical axis, the material absorptance varies only by $2 \%$, whereas the beam trapping effect increases the absorptance by $60 \%$ in our case.

\section{B. Application to a single track}

The same investigation is carried out for a laser single track, with a power of $300 \mathrm{~W}$, a $1 / e^{2}$ spot size of $140 \mu \mathrm{m}$ (i.e., $I=2 \mathrm{MW} / \mathrm{cm}^{2}$ ), and a scan speed of $700 \mathrm{~mm} / \mathrm{s}$. Note that for numerical stability reasons, a power ramping of $150 \mu$ s was implemented. Such additional element will be taken into consideration for the following discussion. Again, an image sequence of the keyhole formation is given in Figs. 7(a)-7(e), and the associated keyhole depth and absorptance over time are given in Fig. 8. Here, the similarity with the previous stationary case is notable. The same fusion regimes preceding keyhole formation are observed, and there is a high correlation between the keyhole depth and absorptance over time (Fig. 8). However, the scanning configuration has some specificities that should be discussed.

First, the conduction and forced conduction steps are very similar to the static case [Fig. 7(a)]. The incident laser irradiation is reflected outward, so the absorptance keeps its initial value (here we have kept the value of 0.39) (Fig. 8). Contrary to the static case, when the vapor depression is generated, the "drilling" process is not strictly vertical, but normal to the depression front wall which is inclined due to the welding speed. Consequently, the melt flow (located under the vapor depression and at its sides) is directed rearward the melt pool. The ejection velocity is initially very close to the scanning speed $(0.7 \mathrm{~m} / \mathrm{s})$ and progressively increases as the depression deepen, because the section through which the melted metal flows reduces. For instance, at keyhole threshold $(t=250 \mu \mathrm{s})$, the maximum velocity magnitude is about $2.5 \mathrm{~m} / \mathrm{s}$, and at the end of the simulation, it reaches about $8 \mathrm{~m} / \mathrm{s}$. Note that the ejection velocity determines the position of the vapor depression rear wall and, indirectly, the keyhole threshold. Indeed, the higher the ejection velocity, the further the depression rear wall open ${ }^{42}$ (i.e., the greater is the depression aperture). Consequently, the incident power necessary to create a keyhole is all the higher. 
(a)

(b)

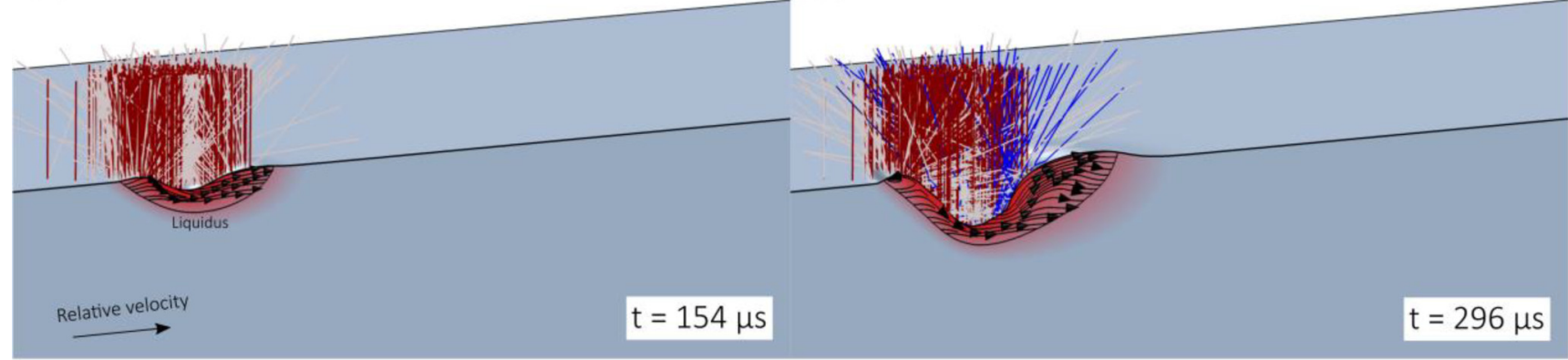

(c)

(d)
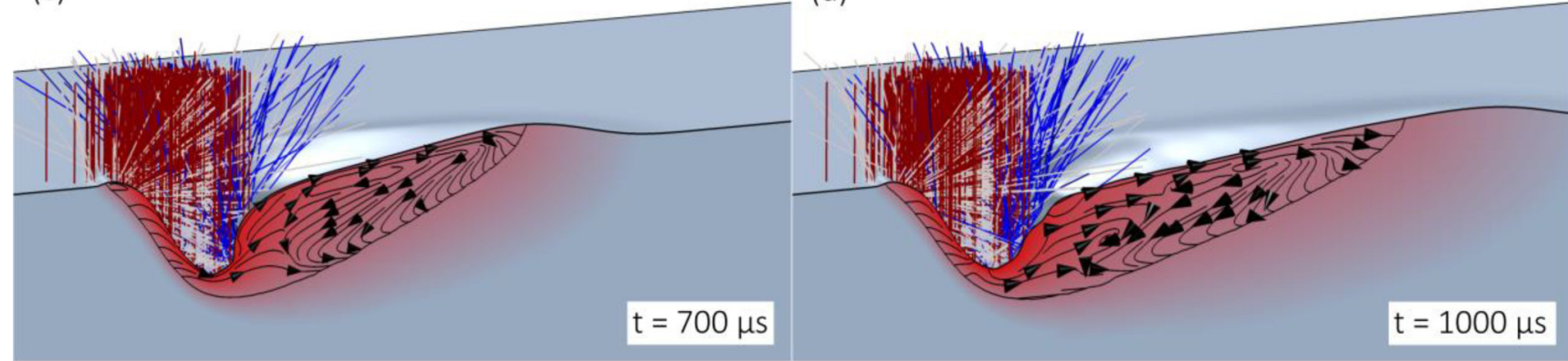

(e)
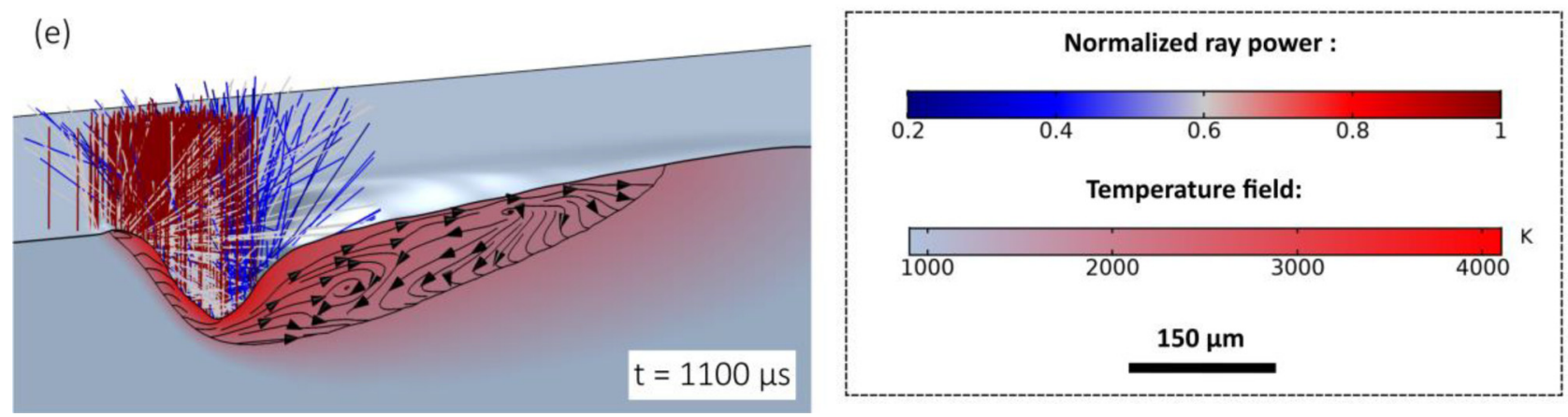

FIG. 7. Keyhole formation steps during a single track. (a) Vapor depression formation. (b) Transition between vapor depression and keyhole. (c) Keyhole formation. [(d) and (e)] Propagation of melt pool corrugations. X-ray images were reprinted with permission from R. Cunningham, C. Zhao, N. Parab, C. Kantzos, J. Pauza, K. Fezzaa, T. Sun, and A. D. Rollett, Science 363, 849-852 (2019). Copyright 2019, AAAS.

In the transient regime, the equilibrium between the keyhole penetration and opening rates also determines the instant at which the keyhole threshold is reached. Here, this event occurs at $t=250 \mu \mathrm{s}$ [Fig. 7(b)]. The fraction of the rays that are reflected downward contributes to the keyhole penetration rate and makes it deviating from the estimation made without RT (Fig. 8). These rays then go out of the keyhole after three or four reflections, with a residual power that lies between 0.2 and 0.3 times the incident power [Fig. 7(b)]. The fraction of the rays that are reflected horizontally contributes to heat the keyhole rear front and to make it fluctuate under the action of the recoil pressure. Both the keyhole depth and absorptance then fluctuate accordingly (Fig. 8). Here, the process is similar to the static case, except that the transition between the vapor depression and keyhole occurs more continuously. This smooth transition must be reinforced by the implementation of a power ramping in our model.

As the keyhole deepens, the front wall tilts further and more rays are reflected downward, contributing to the keyhole penetration [Fig. 7(c)]. However, at the same time, heat lost by conduction in the solid increases proportionally. ${ }^{42}$ As a result, the keyhole penetration rate slows down and the absorptance reaches a plateau. At this stage, a relative equilibrium is found between the keyhole penetration and opening rates such that oscillations of absorptance attenuate (Fig. 8). Such absorptance plateau is also observed in Tang's model, ${ }^{44}$ but with less attenuation. Additionally, frequency and amplitude change during laser single tracks have been observed 


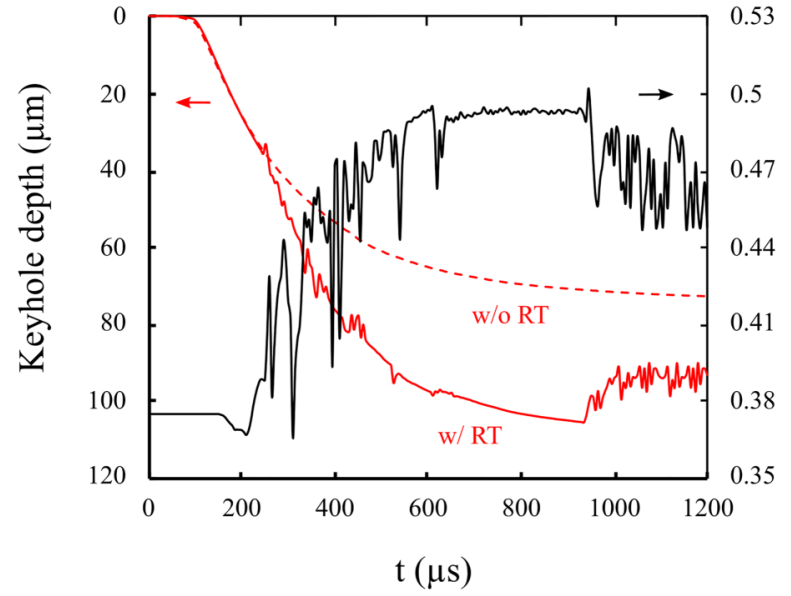

FIG. 8. Keyhole depth (with and without RT) and absorptance over time in the scanning mode.

experimentally by Simonds et al., ${ }^{14}$ especially in the transition between the forced conduction and the keyhole regimes. However here, we believe that the oscillations are further attenuated by numerical damping inherent to our model method. Nevertheless, at this stage, the keyhole rear wall is almost vertical and is consequently mainly irradiated by the secondary reflected rays [Fig. 7(c)]. As the keyhole rear front continues to widen (as the hydrodynamics is not established yet), under the action of the recoil pressure, the keyhole opens. Consequently, the absorptance decreases and so is the keyhole depth. The equilibrium previously found is then lost, the keyhole oscillates, generating corrugations at the bottom of the keyhole rear wall [Fig. 7(d)], which propagates rearward the melt pool [Fig. 7(e)]. This feature is clearly visible on some $\mathrm{x}$-ray images given by Cunningham et al. ${ }^{3}$ and is well reproduced by our model (Fig. 9).

Additionally, we have compared the keyhole depth and width (at mid-depth as in Ref. 25) obtained numerically and experimentally at the steady state, in comparable conditions. The results are

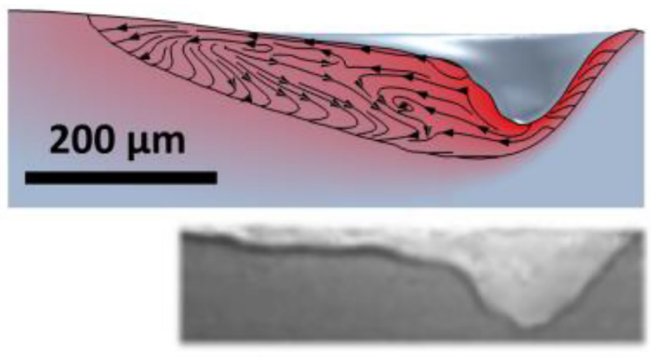

FIG. 9. Comparison of FE and experimental keyhole shapes. X-ray images were reprinted with permission from R. Cunningham, C. Zhao, N. Parab, C. Kantzos, J. Pauza, K. Fezzaa, T. Sun, and A. D. Rollett, Science 363, 849-852 (2019). Copyright 2019, AAAS.
TABLE II. Comparison of keyhole dimensions obtained numerically and experimentally.

\begin{tabular}{lccc}
\hline \hline & $\begin{array}{c}I \\
\left(\mathrm{MW} / \mathrm{cm}^{2}\right)\end{array}$ & $\begin{array}{c}\text { Depth } \\
(\mu \mathrm{m})\end{array}$ & $\begin{array}{c}\text { Width at } 1 / 2 \text { depth } \\
(\mu \mathrm{m})\end{array}$ \\
\hline FEM & 2.0 & 94 & 105 \\
XP & 2.4 & $\sim 110$ & $\sim 130$ \\
$\epsilon_{\%}$ & $16 \%$ & $15 \%$ & $20 \%$ \\
\hline \hline
\end{tabular}

summarized in Table II. Note that the chosen process conditions are not strictly identical, but the difference between the initial incident intensities is reflected in the same proportions in the keyhole dimensions. Furthermore, according to Ref. 5, the keyhole front wall inclination at the steady state can be estimated by

$$
\tan \theta \approx D_{0} / e
$$

where $\theta$ is the inclination angle relative to the vertical axis and $e$ is its penetration depth.

Here, the measured inclination of $56^{\circ}$ agrees with the estimation given by Eq. (16), i.e., $\operatorname{atan}(140 / 94)=56.1^{\circ}$.

Finally, the presented FE model provides encouraging results that agree well with most recent $\mathrm{x}$-ray images, both qualitatively and quantitatively. Notice, however, that this tool is very computationally expensive. For this reason, if it is well adapted for research and fundamental understanding, it is in the present state not ready for direct industrial use. However, such model methods could benefit from progress made in GPU computing that could help to make multiphysical models more accessible in the near future. ${ }^{45}$

\section{CONCLUSIONS AND PROSPECTS}

In summary, a finite element model of laser-induced keyhole has been developed, taking into account the melt pool hydrodynamics (surface tension, Marangoni shear stress, and recoil pressure) and a ray-tracing algorithm to compute self-consistently the absorbed power as a function of the keyhole geometry. We used it to explore keyhole formation mechanisms through two case studies: a static illumination case and a laser single track. We have observed that keyhole formation is characterized by a complex interaction between the melt pool hydrodynamics and the ray optics, which must be accounted for to understand the transient dynamics of keyhole formation mechanisms. Particularly, in the stationary illumination mode, we have observed that

(1) Keyhole forms in five steps: (1) conduction melting, (2) vapor depression formation, (3) vapor depression instability, (4) keyhole formation, and (5) chaotic keyhole fluctuations.

(2) The second stage constitutes a third melting mode in welding/ LPBF, between the conduction and the keyhole modes. This "forced conduction" regime is characterized by a semicircular melt pool shape (like in conduction melting), but its depth and its energy balance are determined by the action of the recoil pressure on the melt. However, it is not a keyhole because the incident laser irradiation is absorbed in the same proportions as in the conduction mode. 
(3) Transition between the third and fourth stages occurs very sharply when the incident laser irradiation is focused by the melt pool toward the laser optical axis. Locally, the absorbed intensity increases by at least 1 order of magnitude.

(4) Fluctuations of the vapor depression/keyhole result from the redistribution of the absorbed intensity because of multiple reflections.

(5) There is an evident correlation between the keyhole depth and the melt pool absorptivity.

In the scanning laser configuration, the keyhole steps are very similar. There is also a high correlation between the keyhole depth and the melt pool absorptivity, and the destabilization mechanisms are identical. However, we have also highlighted that

(1) In transient conditions, while the welding speed is constant, the keyhole depth reaches a maximum before decreasing toward its stationary value.

(2) Accordingly, the keyhole absorptance reaches a maximum before decreasing to its steady state value.

Globally, the FE model presents good similarities with the experiments, both qualitatively and quantitatively. However, to provide a fuller picture of the keyhole formation mechanisms, future work should be dedicated to exploring more process conditions and different materials, particularly with very reflective ones (such as aluminum or copper). Similarly, the combined effects of the welding speed and the incident laser power should be more systematically investigated. Finally, note that in the present model, we did not consider the possible interaction between the keyhole and the vapor plume. Conclusions on keyhole stabilization mechanisms should also take this aspect into account in the future.

\section{ACKNOWLEDGMENTS}

The authors are grateful to Anthony D. Rollett and Tao Sun for helpful discussion on their x-ray experiments. This work has been supported by Safran Additive Manufacturing and Association Nationale de la Recherche et de la Technology (ANRT).

\section{REFERENCES}

'I. Yadroitsev, Selective Laser Melting: Direct Manufacturing of 3D-Objects by Selective Laser Melting of Metal Powders (Lambert Academic Publishing, Saarbrücken, 2009).

${ }^{\mathbf{2}}$ R. Fabbro, "Scaling laws for the laser welding process in keyhole mode," J. Mater. Process. Technol. 264, 346-351 (2019).

${ }^{3}$ R. Cunningham, C. Zhao, N. Parab, C. Kantzos, J. Pauza, K. Fezzaa, T. Sun, and A. D. Rollett, "Keyhole threshold and morphology in laser melting revealed by ultrahigh-speed x-ray imaging," Science 363, 849-852 (2019).

${ }^{4}$ Y. Zhang, G. Chen, H. Wei, and J. Zhang, "A novel "sandwich" method for observation of the keyhole in deep penetration laser welding," Opt. Lasers Eng. 46, 133-139 (2008).

${ }^{\mathbf{5}}$ R. Fabbro, "Melt pool and keyhole behaviour analysis for deep penetration laser welding," J. Phys. D Appl. Phys. 43, 445501 (2010).

${ }^{6}$ Vladimir S. Golubev, "Laser welding and cutting: recent insights into fluiddynamics mechanisms," Proc. SPIE 5121, Laser Processing of Advanced Materials and Laser Microtechnologies (2 September 2003).

${ }^{7} \mathrm{P}$. Berger, H. Hügel, and T. Graf, "Understanding pore formation in laser beam welding," Phys. Procedia 12, 241-247 (2011).

${ }^{8}$ A. Matsunawa, J.-D. Kim, N. Seto, M. Mizutani, and S. Katayama, "Dynamics of keyhole and molten pool in laser welding," J. Laser Appl. 10, 247-254 (1998).
${ }^{9}$ M. H. Cho, D. Farson, J. Y. Lee, and C. D. Yoo, “Laser weld keyhole dynamics," in International Congress on Applications of Lasers \& Electro-Optics (Laser Institute of America, Jacksonville, FL, 2001), pp. 925-932.

${ }^{10}$ C. Zhao, Q. Guo, X. Li, N. Parab, K. Fezzaa, W. Tan, L. Chen, and T. Sun, "Bulk-explosion-induced metal spattering during laser processing," Phys. Rev. X 9, 021052 (2019).

${ }^{11}$ A. A. Martin, Aiden A. Martin, Nicholas P. Calta, Joshua A. Hammons, Saad A. Khairallah, Michael H. Nielsen, Richard M. Shuttlesworth, Nicholas Sinclair, Manyalibo J. Matthews, Jason R. Jeffries, Trevor M. Willey, and Jonathan R. I. Lee, "Ultrafast dynamics of laser-metal interactions in additive manufacturing alloys captured by in situ X-ray imaging," Mater. Today Adv. 1, 100002 (2019).

${ }^{12}$ M. Miyagi and J. Wang, "Keyhole dynamics and morphology visualized by in-situ X-ray imaging in laser melting of austenitic stainless steel," J. Mater. Process. Technol. 282, 116673 (2020).

${ }^{13}$ J. Trapp, A. M. Rubenchik, G. Guss, and M. J. Matthews, "In situ absorptivity measurements of metallic powders during laser powder-bed fusion additive manufacturing," Appl. Mater. Today 9, 341-349 (2017).

${ }^{14}$ B. J. Simonds, J. Sowards, J. Hadler, E. Pfeif, B. Wilthan, J. Tanner, C. Harris, P. Williams, and J. Lehman, "Time-resolved absorptance and melt pool dynamics during intense laser irradiation of a metal," Phys. Rev. Appl. 10, 044061 (2018).

${ }^{15}$ Xudong Zhang, Wuzhu Chen, Jialie Ren, Guoqing Huang, and Hongjun Zhang, "Laser welding mode transition and influence of thermal focusing on mode transition," Proc. SPIE 2888, Laser Processing of Materials and Industrial Applications (30 September 1996).

${ }^{16}$ V. Bruyere, C. Touvrey, P. Namy, and N. Authier, "Multiphysics modeling of pulsed laser welding,” J. Laser Appl. 29, 022403 (2017).

${ }^{17}$ D. Grange, A. Queva, G. Guillemot, M. Bellet, J.-D. Bartout, and C. Colin, "Effect of processing parameters during the laser beam melting of a nickel based alloy: Comparison between simulated and experimental melt pool shape," J. Mater. Process. Technol. 289, 116897 (2021).

${ }^{18}$ H. Ki, P. S. Mohanty, and J. Mazumder, "Modelling of high-density lasermaterial interaction using fast level set method," J. Phys. D Appl. Phys. 34, 364-372 (2001).

${ }^{19}$ J. Y. Lee, S. H. Ko, D. F. Farson, and C. D. Yoo, "Mechanism of keyhole formation and stability in stationary laser welding," J. Phys. D Appl. Phys. 35, 1570-1576 (2002).

${ }^{20} \mathrm{M}$. Medale, C. Touvrey, and R. Fabbro, "An axi-symmetric thermo-hydraulic model to better understand spot laser welding," Eur. J. Comput. Mech. 17, 795-806 (2008).

${ }^{\mathbf{2 1}} \mathrm{W}$. Tan and Y. C. Shin, "Analysis of multi-phase interaction and its effects on keyhole dynamics with a multi-physics numerical model," J. Phys. D Appl. Phys. 47, 345501 (2014).

${ }^{22}$ S. Pang, X. Chen, J. Zhou, X. Shao, and C. Wang, “3D transient multiphase model for keyhole, vapor plume, and weld pool dynamics in laser welding including the ambient pressure effect," Opt. Lasers Eng. 74, 47-58 (2015).

${ }^{23}$ M. Bayat, S. Mohanty, and J. H. Hattel, "Multiphysics modelling of lack-of-fusion voids formation and evolution in IN718 made by multi-track/ multi-layer L-PBF," Int. J. Heat Mass Transfer 139, 95-114 (2019).

${ }^{24}$ J. Ye, S. A. Khairallah, A. M. Rubenchik, M. F. Crumb, G. Guss, J. Belak, and M. J. Matthews, "Energy coupling mechanisms and scaling behavior associated with laser powder bed fusion additive manufacturing," Adv. Eng. Mater. 21, 1900185 (2019).

${ }^{25}$ N. Kouraytem, X. Li, R. Cunningham, C. Zhao, N. Parab, T. Sun, A. D. Rollett, A. D. Spear, and W. Tan, "Effect of laser-matter interaction on molten pool flow and keyhole dynamics," Phys. Rev. Appl. 11, 064054 (2019).

${ }^{26}$ M. Courtois, M. Carin, P. Le Masson, S. Gaied, and M. Balabane, "Complete heat and fluid flow modeling of keyhole formation and collapse during spot laser welding," in International Congress on Applications of Lasers \& Electro-Optics, Miami, FL, USA (Laser Institute of America, 2013), pp. 77-84.

${ }^{27}$ Y. A. Mayi, M. Dal, P. Peyre, C. Metton, C. Moriconi, and R. Fabbro, “An original way of using $\mathrm{COMSOL}^{\star}$ application builder to enhance multiphysical 
simulation of laser welding processes," in Proceedings of the COMSOL Conference 2020, Europe, 14-15 October 2020 (COMSOL AB, Stockholm, Sweden, 2020).

${ }^{28}$ C. Bonacina, G. Comini, A. Fasano, and M. Primicerio, "Numerical solution of phase-change problems," Int. J. Heat Mass Transfer 16, 1825-1832 (1973).

${ }^{29} \mathrm{C}$. Mas, Modélisation physique du procédé de découpe de métaux par laser (Université Pierre et Marie Curie, Paris VI, Paris, 2003).

${ }^{30} \mathrm{C}$. J. Knight, "Theoretical modeling of rapid surface vaporization with back pressure," AIAA J. 17, 78-1220 (1979).

${ }^{31}$ V. R. Voller and C. Prakash, "A fixed grid numerical modelling methodology for convection-diffusion mushy region phase-change problems," Int. J. Heat Mass Transfer 30, 1709-1719 (1987).

${ }^{32}$ COMSOL, Multiphysics ${ }^{\otimes} v 5.5$ (COMSOL AB, Stockholm, 2020).

${ }^{33}$ K. C. Mills, Recommended Values of Thermophysical Properties for Selected Commercial Alloys (Woodhead, Cambridge, 2002).

${ }^{34} \mathrm{P}$. Johnson and R. Christy, "Optical constants of transition metals: Ti, V, Cr, Mn, Fe, Co, Ni, and Pd,” Phys. Rev. B 9, 5056-5070 (1974).

${ }^{35}$ Lange's Handbook of Chemistry, edited by J. A. Dean and N. A. Lange, 15th ed. (McGraw-Hill, New York, NY, 1999).

${ }^{36} \mathrm{~K}$. Zhou and B. Wei, "Determination of the thermophysical properties of liquid and solid Ti-6Al-4V alloy,” Appl. Phys. A 122, 248 (2016).

${ }^{37}$ H. Kwon, W.-K. Baek, M.-S. Kim, W.-S. Shin, and J. J. Yoh, "Temperature-dependent absorptance of painted aluminum, stainless steel 304, and titanium for $1.07 \mu \mathrm{m}$ and $10.6 \mu \mathrm{m}$ laser beams," Opt. Lasers Eng. 50, 114-121 (2012).

${ }^{38}$ T. R. Allen, W. Huang, J. R. Tanner, W. Tan, J. M. Fraser, and B. J. Simonds, "Energy-Coupling mechanisms revealed through simultaneous keyhole depth and absorptance measurements during laser-metal processing," Phys. Rev. Appl. 13, 064070 (2020).

${ }^{39}$ Y. A. Mayi, M. Dal, P. Peyre, M. Bellet, C. Metton, C. Moriconi, and R. Fabbro, "Laser-induced plume investigated by finite element modelling and scaling of particle entrainment in laser powder bed fusion," J. Phys. D Appl. Phys. 53, 075306 (2020).
${ }^{40} \mathrm{X}$. Li, C. Zhao, T. Sun, and W. Tan, "Revealing transient powder-gas interaction in laser powder bed fusion process through multi-physics modeling and highspeed synchrotron X-ray imaging," Addit. Manuf. 35, 101362 ( 2020).

${ }^{41} \mathrm{~V}$. Semak and A. Matsunawa, "The role of recoil pressure in energy balance during laser materials processing," J. Phys. D Appl. Phys. 30, 2541-2552 (1997).

${ }^{42} \mathrm{R}$. Fabbro, "Depth dependence and keyhole stability at threshold, for different laser welding regimes," Appl. Sci. 10, 1487 (2020).

${ }^{43}$ A. Gouffé, "Corrections d'ouverture des corps noirs artificiels compte tenu des diffusions multiples internes," Rev. Opt. 24, 1-10 (1945).

${ }^{44}$ C. Tang, K. Q. Le, and C. H. Wong, "Physics of humping formation in laser powder bed fusion," Int. J. Heat Mass Transfer 149, 119172 (2020).

${ }^{45}$ Dmitry S. Nakapkin, Andrey V. Zakirov, Sergei A. Belousov, Maria V. Bogdanova, Boris A. Korneev, Andrey E. Stepanov, Anastasia Y. Perepelkina, Vadim D. Levchenko, Boris V. Potapkin, and Andrey Meshkov, "Finding optimal parameter ranges for laser powder bed fusion with predictive modeling at mesoscale," in Paper Presented at the II International Conference on Simulation for Additive Manufacturing-Sim-AM 2019, Pavia, Italy, 11-13 September 2019 (International Centre for Numerical Methods in Engineering (CIMNE), Barcelona, Spain, 2019).

\section{Meet the Authors}

Yaasin Mayi is a Ph.D. candidate at both Safran Additive Manufacturing and PIMM Laboratory, with previous Industrial Engineering studies at Art et Metiers Institute of Technology (France) and MSc in Advanced Materials at Cranfield University (UK). He is currently developing numerical models to investigate several physical phenomena occurring in Laser Powder Bed Fusion (LBPF), specifically laser-induced vaporization and its consequences on melt pool and vapor plume dynamics. 